\title{
Endothelial Dysfunction in Systemic Lupus Erythematosus: Evaluation with ${ }^{13} \mathrm{~N}$-Ammonia PET
}

\author{
Erick Alexánderson ${ }^{1,2}$, Juan M. Ochoa ${ }^{2}$, Rodrigo Calleja ${ }^{2}$, Juan G. Juárez-Rojas ${ }^{3}$, John O. Prior ${ }^{4}$, Rodrigo Jácome ${ }^{2}$, \\ Edgar Romero $^{2}$, Aloha Meave ${ }^{1,2}$, and Carlos Posadas-Romero ${ }^{3}$ \\ ${ }^{1}$ Department of Nuclear Cardiology and Cardiac Imaging, Instituto Nacional de Cardiología "Ignacio Chávez,” México City, \\ México; ${ }^{2}$ PET/CT Cyclotron Unit, Facultad de Medicina, UNAM, México City, México; ${ }^{3}$ Department of Endocrinology, Instituto \\ Nacional de Cardiología "Ignacio Chávez," México City, México; and ${ }^{4}$ Department of Nuclear Medicine, Centre Hospitalier \\ Universitaire Vaudois et Université de Lausanne, Lausanne, Switzerland
}

Systemic lupus erythematosus (SLE) affects multiple organs and systems, severely involving the cardiovascular system. The aim of this study was to evaluate the presence of endothelial dysfunction with ${ }^{13} \mathrm{~N}$-ammonia PET in asymptomatic SLE patients. Methods: We enrolled 16 women with SLE and 16 healthy women. Myocardial blood flow (MBF) was quantified in a 64-slice PET/CT scanner at rest, during a cold pressor test (CPT), and during stress. Endothelium-dependent vasodilation index, \% $\triangle \mathrm{MBF}$, and myocardial flow reserve (MFR) were calculated. Results: There were 16 women in the SLE group (mean age $\pm \mathrm{SD}, 31.4 \pm 8.3 \mathrm{y}$ ) and 16 women in the healthy control group $(31.5 \pm 11.1 \mathrm{y})$. Mean endothelium-dependent vasodilatation index and $\% \triangle \mathrm{MBF}$ were significantly lower in SLE patients $(1.18 \pm 0.55$ vs. $1.63 \pm 0.65, P=0.04$, and $18 \pm 55$ vs. $63 \pm 65, P=0.04$, respectively). MFR was also lower in the SLE group ( $2.41 \pm 0.59$ vs. $2.73 \pm 0.77, P=0.20)$. Conclusion: SLE patients who are free of active disease present abnormal coronary flow and endothelial dysfunction. It is necessary to develop and intensify treatment strategies directed to CAD in SLE patients.

Key Words: ${ }^{13} \mathrm{~N}$-ammonia PET; endothelium-dependent vasodilatation index; endothelial dysfunction; MFR; systemic lupus erythematosus

J Nucl Med 2010; 51:1927-1931

DOI: 10.2967/jnumed.110.078212

O disease that affects multiple organs and systems and in which the cardiovascular system is severely involved; myocardial infarction and stroke constitute the main cause of death (1). In one study, after the Framingham risk factors for coronary heart disease had been taken into account, patients with SLE showed a 10- and 17-fold increase in

Received Apr. 15, 2010; revision accepted Sep. 8, 2010.

For correspondence or reprints contact: Erick Alexánderson Rosas, Department of Nuclear Cardiology, Instituto Nacional de Cardiología "Ignacio Chavez," Juan Badiano \#1, Col. Seccion XVI, Tlalpan, Mexico City, D.F. 14080, Mexico.

E-mail: alexanderick@yahoo.com

COPYRIGHT @ 2010 by the Society of Nuclear Medicine, Inc. nonfatal myocardial infarction and death due to coronary heart disease, respectively (2).

Various studies have shown that patients with SLE have a higher prevalence of classic cardiovascular risk factors (3). Besides, there are SLE-related risk factors, such as steroid therapy (4). Despite various explanations citing vascular damage as a cause of accelerated atherosclerosis, a specific cause has not been highlighted. However, SLE itself has been shown to represent an independent risk factor for coronary heart disease secondary to endothelial dysfunction (5).

Endothelial dysfunction represents the inability of the endothelium to appropriately modulate myocardial blood flow (MBF) by the secretion of nitric oxide and is considered an early marker of atherosclerosis (6). Endothelial dysfunction has been demonstrated in SLE using brachial artery Doppler ultrasound (7). However, such measurements are unable to detect subclinical atherosclerosis. Coronary microvascular dysfunction has previously been detected with PET in patients with rheumatoid arthritis and SLE by assessing the integrated vasodilator capacity (myocardial flow reserve [MFR], an endothelium-independent index of coronary function (8)) but not by assessing endothelium-dependent coronary vasomotor function (a more reliable index of endothelial dysfunction (9)). Therefore, the current study might have been the first that evaluated both cardiac disease-dependent and -independent coronary endothelial function by quantifying MBF in absolute terms with ${ }^{13} \mathrm{~N}-$ ammonia PET in SLE patients.

The aim of this study was, then, to use ${ }^{13} \mathrm{~N}$-ammonia PET to evaluate the presence of endothelial dysfunction in asymptomatic, uncomplicated SLE patients who were under pharmacologic treatment.

\section{MATERIALS AND METHODS}

\section{Study Population}

Sixteen women diagnosed with SLE were enrolled in this study. All were 18 y old or older. Throughout the study, each patient was taking low-dose steroids $(56.2 \%)$ or antimalarial drugs $(87.5 \%)$. All fulfilled the American College of Rheumatology Criteria for the disease and had been diagnosed with SLE for $7 \pm 4.5 \mathrm{y}$. At the 
time of the study they were asymptomatic, showing no evidence of active disease as assessed by the MEX-SLEDAI Disease Activity Index (10). According to that index, a score greater than 5 is considered to indicate active disease, and our group of SLE patients had a mean score of 2.45. The control group consisted of 16 agematched healthy women without clinical evidence of lupus or any other disease. Because hormonal fluctuations of the menstrual cycle are known to affect coronary flow regulation, the analysis was performed within the first $8 \mathrm{~d}$ of the patient's menstrual cycle. Exclusion criteria included previous myocardial infarction demonstrated by electrocardiography, a history of chest pain, diabetes mellitus, hypertension, cerebral vascular disease, use of oral contraceptives, smoking or drug abuse, known hypersensitivity to any of the substances used during the study, and pregnancy or lactation at the time of the study. Patients were instructed to fast 8-12 h before the test and to avoid food or drinks containing methyl xanthenes for more than $48 \mathrm{~h}$ before the study. Medical history, physical examination, and laboratory examinations were obtained before the PET study. The ethics committee of the Instituto Nacional de Cardiología "Ignacio Chávez" approved the protocol, and each patient gave written consent before participating in the study.

\section{Image Acquisition}

MBF was quantified at rest and during a cold pressor test (CPT), as well as during adenosine-induced hyperemia, using dynamic ${ }^{13} \mathrm{~N}$ ammonia PET/CT in a 64-slice scanner (Biograph TruePoint; Siemens). All data were acquired in list-mode format (11).

After the first intravenous administration of ${ }^{13} \mathrm{~N}$-ammonia (740 $\mathrm{MBq})$, resting serial transaxial dynamic images were acquired in a sequence of 16 image frames $(12 \times 10 \mathrm{~s}, 2 \times 30 \mathrm{~s}, 1 \times 60 \mathrm{~s}$, and $1 \times$ $900 \mathrm{~s})$ (12). The CPT was performed $30 \mathrm{~min}$ later by immersing the left hand of the patient in ice-cold water $\left(4^{\circ} \mathrm{C}\right)$ for $2 \mathrm{~min}$. The ${ }^{13} \mathrm{~N}$-ammonia (740 MBq) injection and PET sequence were initiated $1 \mathrm{~min}$ into the hand immersion. Finally, $30 \mathrm{~min}$ after the end of CPT, a $140 \mu \mathrm{g} / \mathrm{kg} / \mathrm{min}$ intravenous infusion of adenosine was administered over 6 min. ${ }^{13} \mathrm{~N}$-ammonia (740 MBq) was administered at the end of the second minute of the adenosine-infusion phase, and serial images were recorded in the same sequence. Vital signs and a 12-lead electrocardiogram were monitored continuously throughout the study. The acquisition protocol is depicted in Figure 1.

\section{Image Processing}

Regional MBF was quantified for the rest phase and, separately, for the adenosine-stress phase. The sinograms were corrected for attenuation and reconstructed on a dedicated workstation using standard iterative algorithms. The images were analyzed using the PMOD software package (version 3.0, PMOD Technologies Ltd.). Myocardial images were then generated directly from the dynamic ${ }^{13} \mathrm{~N}$-ammonia study, using the last $900-\mathrm{s}$ image. After the myocardial images had been reoriented along the heart axis to form vertical long-axis, horizontal long-axis, and short-axis slices, regions of interest were drawn within the left ventricle on 4-6 consecutive image planes and were projected onto the dynamic ${ }^{13} \mathrm{~N}$-ammonia images to generate blood time-activity curves (input function) and onto the right ventricle to correct for spillover of the septum. Similarly, myocardial regions of interest were drawn on the short-axis slices within the left ventricular myocardium for at least 12 consecutive image planes and were projected onto the dynamic ${ }^{13} \mathrm{~N}$-ammonia images to obtain tissue-activity curves. Time-activity curves were used to calculate mean MBF from the first 12 serially acquired, short-axis slices after myocardial regions of interest had been assigned to the myocardial territories of the left anterior descending, left circumflex, and right coronary arteries on 3 midventricular slices. Both arterial and tissue-activity curves were corrected for physical decay and fitted to a previously validated 2-compartment tracer kinetic model to give values of regional and global $\mathrm{MBF}(\mathrm{mL} / \mathrm{min} / \mathrm{g})$, as previously described (13).

\section{Data Interpretation}

Mean values of global $\mathrm{MBF}$ are given in $\mathrm{mL} / \mathrm{min} / \mathrm{g}$ of myocardium. Rest and CPT MBF were corrected for their corresponding rate-pressure product (RPP) using the following equation: (MBF/ $\mathrm{RPP}) \times 10^{4}(14)$. Endothelium-dependent vasodilatation index (MBF during CPT/MBF at rest), \% $\mathrm{MBF}(\% \Delta \mathrm{MBF}$ of CPT from rest), and MFR (stress MBF/rest MBF) were also calculated using RPP-corrected values. The $\triangle \mathrm{MBF}$ (difference in flow between CPT and baseline) was calculated using uncorrected MBF values, because they have been shown to be independent of the baseline RPP (15).

\section{Statistical Analysis}

Results from the SLE group were compared with those from the healthy control group.

Endothelium-dependent vasodilatation index, MFR, \% $\triangle \mathrm{MBF}$, and $\triangle \mathrm{MBF}$ were used to evaluate endothelial dysfunction (16). Values are presented as mean \pm SD and were compared using the Student 2-tailed unpaired $t$ test. Statistical analysis was performed using SPSS, version 17. A $P$ value of less than 0.05 was considered to indicate statistical significance.

\section{RESULTS}

The 16 women in the SLE group had a mean age of $31.4 \pm$ $8.3 \mathrm{y}$. The 16 age-matched women in the healthy control group were $31.5 \pm 11.1$ y old. Study population characteristics are shown in Table 1. During the study, no important electrocardiography changes were observed. The hemodynamic findings, shown in Table 2, highlight the differences in hemodynamic RPP response between the 2 study groups, as demonstrated by the $\delta$-RPP result $(0.37 \pm 0.26$ in the SLE group vs. $0.16 \pm$ 0.20 in the control group, $P=0.01$ ) (Table 2).
FIGURE 1. Dynamic list-mode PET acquisition protocol. CTAC $=\mathrm{CT}$ attenuation correction; $\mathrm{LM}=$ List mode. ${ }^{*}$ Wait time for radionuclide decay.

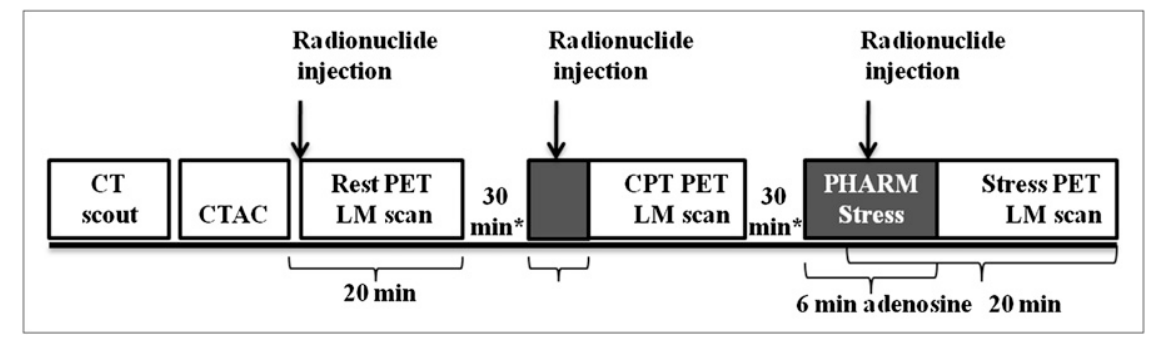


TABLE 1

Study Population Characteristics

\begin{tabular}{|c|c|c|c|}
\hline Characteristic & SLE & Healthy control & $P$ \\
\hline No. of patients & 16 & 16 & - \\
\hline Age $(y)$ & $31.4 \pm 8.3$ & $31.5 \pm 11.1$ & 0.62 \\
\hline Sex, female (\%) & $100 \%$ & $100 \%$ & - \\
\hline $\mathrm{BMI}\left(\mathrm{kg} / \mathrm{m}^{2}\right)$ & $24.8 \pm 3.6$ & $22.2 \pm 1.6$ & 0.52 \\
\hline Fasting glucose (mg/dL) & $87.2 \pm 4.9$ & $77.6 \pm 5.2$ & 0.0001 \\
\hline Total cholesterol (mg/dL) & $130.1 \pm 20.6$ & $159.6 \pm 27.2$ & 0.02 \\
\hline LDL-C (mg/dL) & 67.1. \pm 22.2 & $88.1 \pm 18.4$ & 0.007 \\
\hline $\mathrm{HDL}-\mathrm{C}(\mathrm{mg} / \mathrm{dL})$ & $47.6 \pm 9.4$ & $54.7 \pm 6$ & 0.16 \\
\hline LDL-C/HDL-C index(mg/dL) & 1.4 & 1.6 & 0.43 \\
\hline Triglycerides $(\mathrm{mg} / \mathrm{dL})$ & $107.4 \pm 62.5$ & $118.4 \pm 35.1$ & 0.54 \\
\hline Creatinine (mg/dL) & $0.83 \pm 0.1$ & $0.85 \pm 0.1$ & 0.65 \\
\hline Basal heart rate (beats/min) & $67 \pm 11$ & $70 \pm 6$ & 0.33 \\
\hline Basal blood pressure (systolic/diastolic; $\mathrm{mm} \mathrm{Hg}$ ) & $107 \pm 9 / 74 \pm 7$ & $101 \pm 10 / 68 \pm 5$ & $0.92 / 0.14$ \\
\hline
\end{tabular}

$\mathrm{BMI}=$ Body mass index; HDL-C = high-density lipoprotein cholesterol; LDL-C = low density lipoprotein cholesterol.

Data are mean \pm SD.

Uncorrected and corrected MBF values during rest, CPT, and adenosine stress are shown in Table 3. Individual flow responses from rest to CPT, as well as individual responses to adenosine administration, are shown in Figure 2. Rest MBF was similar between the groups $(0.71 \pm 0.15$ vs. $0.73 \pm$ $0.27, P=0.7)$. Uncorrected CPT MBF was statistically similar $(1.07 \pm 0.33$ vs. $1.45 \pm 0.85, P=0.11)$; however, when corrected for differences in RPP, CPT MBF was significantly lower in the SLE group than in the healthy control group $(1.16 \pm 0.48$ vs. $1.94 \pm 1.15, P=0.01)$. Interestingly, there was a trend toward decreased stress MBF in the SLE group ( $2.34 \pm 0.39$ vs. $2.96 \pm 1.18, P=0.058)$.

Mean endothelium-dependent vasodilatation index, $\% \triangle \mathrm{MBF}, \triangle \mathrm{MBF}$, and MFR are shown in Table 4. Mean endothelium-dependent vasodilatation index and \%

TABLE 2

Hemodynamic Findings in Both Study Groups During Rest, CPT, and Pharmacologic Stress

\begin{tabular}{|c|c|c|c|}
\hline Parameter & SLE & Healthy control & $P$ \\
\hline \multicolumn{4}{|c|}{ Heart rate (beats/min) } \\
\hline Rest & $67 \pm 11$ & $62 \pm 6$ & 0.68 \\
\hline CPT & $81 \pm 20$ & $67 \pm 8$ & 0.01 \\
\hline Stress & $96 \pm 13$ & $88 \pm 11$ & 0.06 \\
\hline \multicolumn{4}{|c|}{ Systolic blood pressure $(\mathrm{mm} \mathrm{Hg})$} \\
\hline Rest & $107 \pm 9$ & $108 \pm 13$ & 0.74 \\
\hline CPT & $120 \pm 8$ & $113 \pm 9$ & 0.03 \\
\hline Stress & $117 \pm 9$ & $112 \pm 10$ & 0.17 \\
\hline \multicolumn{4}{|l|}{ RPP } \\
\hline Rest & $7,201 \pm 1,434$ & $6,628 \pm 833$ & 0.18 \\
\hline CPT & $9,819 \pm 2,584$ & $7,583 \pm 845$ & 0.03 \\
\hline Stress & $11,200 \pm 1,911$ & $9,729 \pm 998$ & 0.01 \\
\hline$\delta$-RPP (\%) & $37 \% \pm 26 \%$ & $16 \% \pm 20 \%$ & 0.01 \\
\hline $\begin{array}{r}\delta-\mathrm{RPP}=\text { perc } \\
\text { (RPP CPT - RPP } \\
\text { Data are mean }\end{array}$ & $\begin{array}{l}\text { age change in R } \\
\text { st)/RPP rest. } \\
\text { SD. }\end{array}$ & between rest a & $\mathrm{CPT}=$ \\
\hline
\end{tabular}

$\triangle \mathrm{MBF}$ were significantly lower in the SLE group than in the healthy control group ( $1.18 \pm 0.55$ vs. $1.63 \pm 0.65$, $P=0.04$, and $18 \pm 55$ vs. $63 \pm 65, P=0.04$, respectively). $\triangle \mathrm{MBF}$ was lower in the SLE group than in the healthy control group, but the difference was not statistically significant $(0.37 \pm 0.35$ vs. $0.71 \pm 0.63, P=0.06)$.

MFR was also lower in the SLE group-lower than the cutoff of normality $(>2.5)$-although the difference between groups was not statistically significant $(P=0.2)$. That being said, if uncorrected rest MBF is used to determine MFR, there is a significant difference between the SLE group and the healthy control group $(4.11 \pm 1.02$ vs. $3.37 \pm 0.64, P=0.02$, respectively) (Table 4 ).

Possible associations between MBF and disease duration, steroid treatment, or disease activity indices were assessed. However, no statistical correlations were found between these variables.

\section{DISCUSSION}

This study compared MBF between a group of SLE patients and a healthy control group. At rest and during $\mathrm{CPT}, \mathrm{MBF}$ depends on left ventricular myocardial work and

TABLE 3

MBF During the 3 Phases of PET Study

\begin{tabular}{cccc}
\hline MBF & SLE & Healthy control & $P$ \\
\hline Rest & & & \\
Uncorrected & $0.71 \pm 0.15$ & $0.73 \pm 0.27$ & 0.73 \\
$\quad$ Corrected & $1.00 \pm 0.17$ & $1.13 \pm 0.43$ & 0.27 \\
CPT & & & \\
$\quad$ Uncorrected & $1.07 \pm 0.33$ & $1.45 \pm 0.85$ & 0.11 \\
$\quad$ Corrected & $1.16 \pm 0.48$ & $1.94 \pm 1.15$ & 0.01 \\
$\quad$ Stress & & & \\
$\quad$ Uncorrected & $2.34 \pm 0.39$ & $2.96 \pm 1.18$ & 0.05
\end{tabular}

Data are mean $\pm \mathrm{SD}$. Corrected MBF $=(\mathrm{MBF} / \mathrm{RPP}) \times 10^{4}$. 


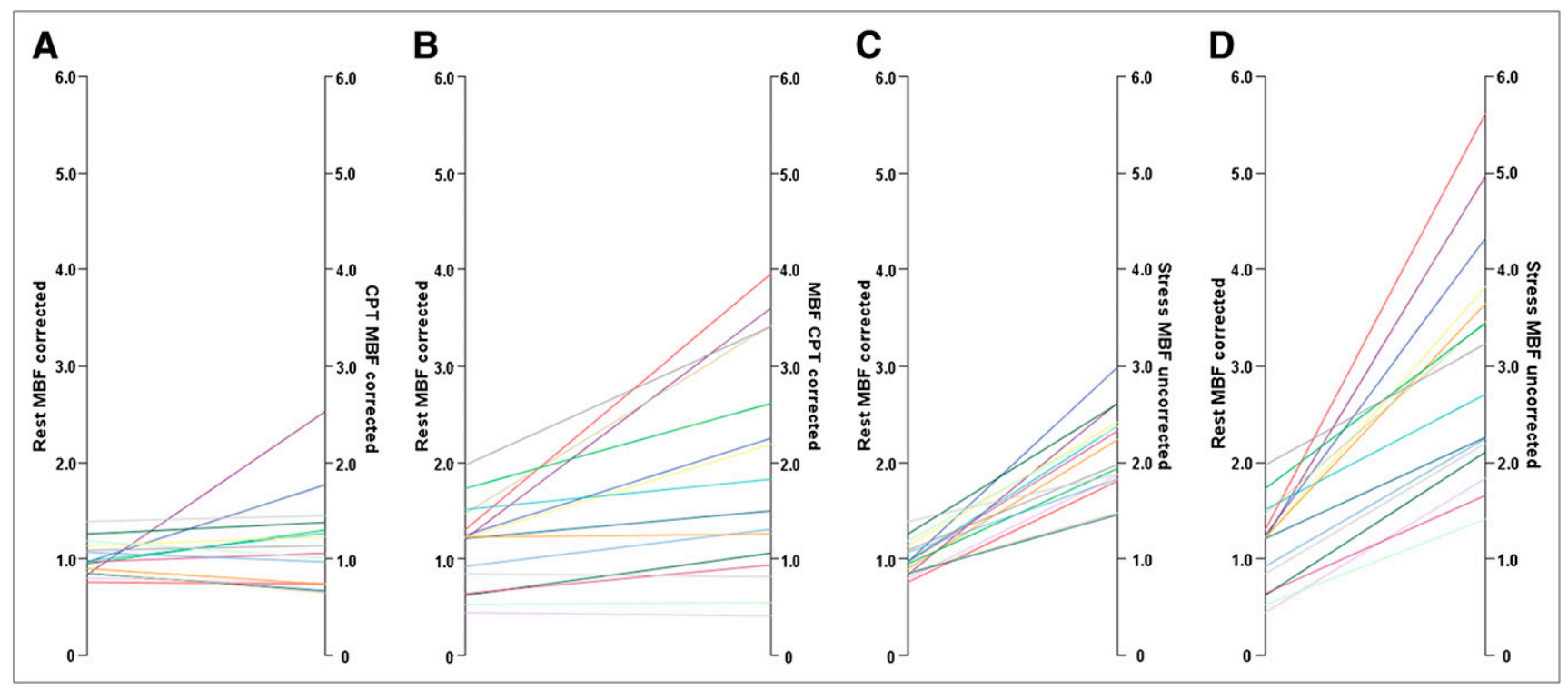

FIGURE 2. Individual MBF response from rest to CPT and stress in both groups. Shown is MBF from rest to CPT in SLE group (A) and healthy control group (B) and MBF from rest to stress in SLE group (C) and healthy control group (D).

thus on the hemodynamic state (17). Therefore, in our study MBF was normalized during rest and CPT to the RPP to confirm that the differences found between groups do reflect endothelial dysfunction and not changes in hemodynamic regulation. In this regard, the difference in the hemodynamic RPP response between groups as shown in Table 2 was significantly different, with a lower response to CPT in healthy controls. A possible reason could be that 6 of the 16 healthy controls were studied at a different time. However, differences in RPP responses to CPT would certainly not explain a decreased response in the SLE patients, compared with the response in healthy controls; in this case, we would rather expect the contrary. The difference could also be explained by differences in response to pain intrinsic to SLE secondary to CPT (18). Besides, despite the greater (though not statistically significant) hemodynamic response in the SLE group, that group had a considerably lower $\triangle \mathrm{MBF}$ (Table 4), which, given these hemodynamic differences, shows this measure to be preferable because less dependent on baseline MBF and RPP.

The assessment of endothelium-dependent coronary vasomotor function targets the response of MBF to $\alpha$-adrenergic stimulation with CPT; a diminished MBF response to CPT is strongly associated with endothelial dysfunction (19). MFR reflects the response of basal MBF to administration of vascular smooth muscle-relaxing agents, reducing the resistance of flow and thus generating an increase in MBF. The total hyperemic response to pharmacologic stress reflects the combined vasodilator effect of vascular smooth muscle and endothelium and is defined as the total integrated vasodilator capacity (9). In our study, we found 2 interesting situations: SLE patients had a statistically lower endothelium-dependent vasodilatation index than did healthy controls, allowing us to affirm that endothelial dysfunction exists, and there was a trend toward a decrease in the total vasodilation capacity in patients with SLE, highlighted by the lower but not statistically significant MFR. In this regard, it is true that about $36 \%$ of the hyperemic response depends on endothelium-dependent vasodilation. However, a decrease in MFR to less than 2.5 was not considered a diagnostic criterion of endothelial dysfunction because such a decrease can also indicate nonsignificant coronary artery disease or mild multivessel disease. Regarding that issue, a coronary anatomy assessment in this population could have strengthened the MFR results.

The endothelium-dependent vasodilatation index and MFR measure different aspects of coronary circulatory function, making it feasible to distinguish whether the

TABLE 4

Endothelial Function Indices

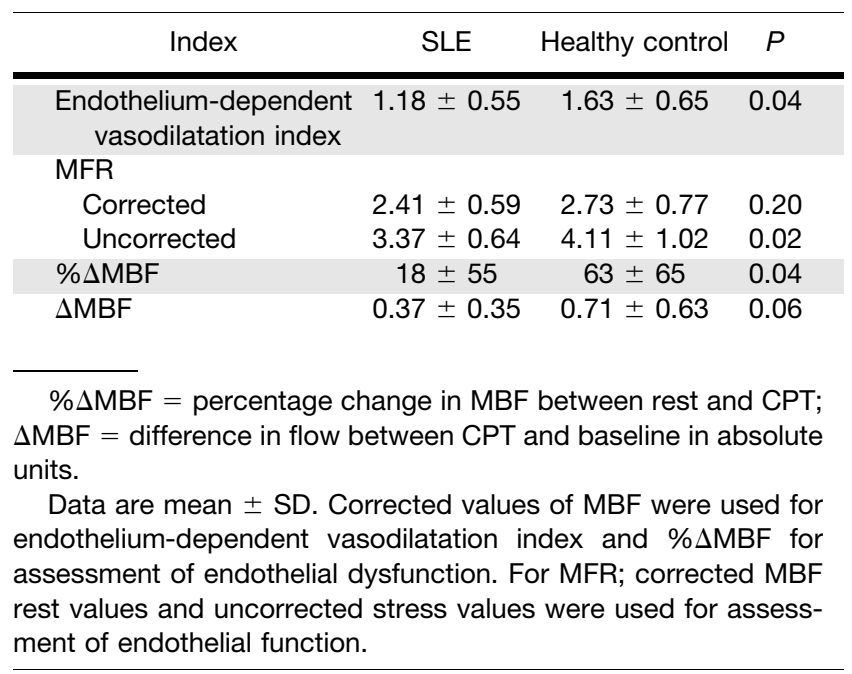


alteration is confined to the endothelium or involves vascular smooth muscle function.

Taking into account these issues, we can assume from this study that SLE patients have both endothelial and smooth muscle dysfunction, the former being more severely involved, as it may be the result of direct damage from microvascular inflammatory disease.

It is known that changes in endothelial dysfunction happen early in the natural history of vascular disease; this study supports such a statement concerning the coronary vessels of patients with SLE. As previously mentioned, ${ }^{13} \mathrm{~N}$-ammonia PET allows detection of vascular changes that could make possible the identification of patients at risk for the progression of vascular disease and provides a means for therapy monitoring. Because the development of major adverse cardiac events and cardiac death is predicted by a low MFR despite normal perfusion (20), it might be important to detect early endothelial dysfunction in patients with SLE and to treat aggregated cardiovascular risk factors early, and the disease per se, thus slowing or halting the development of endothelial dysfunction before progression to atherosclerosis and major adverse cardiac events or cardiac death.

The relevance of this study relies on the identification of endothelial dysfunction in adequately treated SLE patients who do not demonstrate active disease by a validated SLE index. Although the current available treatment of SLE limits disease activity, one can assume that this treatment does not prevent or stop the development of cardiovascular disease. Thus, the need for new treatment strategies directed toward such relatively unattended disease-induced organ damage is evident.

One limitation of this study is the small population. An obvious drawback of PET-measured noninvasive measurement of coronary endothelial dysfunction is the limited availability of the method, and for developing countries, its expense.

\section{CONCLUSION}

Asymptomatic SLE patients who are free of active disease present an abnormal coronary flow as demonstrated by both smooth muscle dysfunction and endothelial dysfunction measured by ${ }^{13} \mathrm{~N}$-ammonia PET. It is necessary to develop and intensify treatment strategies directed toward atherosclerosis in SLE patients.

\section{ACKNOWLEDGMENTS}

We thank Juan Carlos Ceballos Cantu for skillful assistance with data acquisition and collection, and we thank
Andres Sanabria Rodriguez and Jorge Albarran Lopez for technical support.

\section{REFERENCES}

1. Rubin LA, Urowitz MB, Gladman DD. Mortality in systemic lupus erythematosus: the bimodal pattern revisited. $Q J$ Med. 1985;55:87-98.

2. Esdaile JM, Abrahamowicz M, Grodzicky T, et al. Traditional Framingham risk factors fail to fully account for accelerated atherosclerosis in systemic lupus erythematosus. Arthritis Rheum. 2001;44:2331-2337.

3. Bruce IN. 'Not only...but also': factors that contribute to accelerated atherosclerosis and premature coronary heart disease in systemic lupus erythematosus. Rheumatology. 2005;44:1492-1502.

4. Bulkley BH, Roberts WC. The heart in systemic lupus erythematosus and the changes induced in it by corticosteroid therapy: a study of 36 necropsy patients. Am J Med. 1975;58:243-264.

5. El-Magadmi M, Bodill H, Ahmad Y, et al. Systemic lupus erythematosus: an independent risk factor for endothelial dysfunction in women. Circulation. 2004;110:399-404.

6. Bonetti PO, Lerman LO, Lerman A. Endothelial dysfunction: a marker of atherosclerotic risk. Arterioscler Thromb Vasc Biol. 2003;23:168-175.

7. Piper MK, Raza K, Nuttall SL, et al. Impaired endothelial function in systemic lupus erythematosus. Lupus. 2007;16:84-88.

8. Recio-Mayoral A, Mason JC, Kaski JC, et al. Chronic inflammation and coronary microvascular dysfunction in patients without risk factors for coronary artery disease. Eur Heart J. 2009;30:1837-1843.

9. Schelbert HR. Quantification of myocardial blood flow: what is the clinical role? Cardiol Clin. 2009;27:277-289.

10. Guzman J, Cardiel MH, Arce-Salinas A, Sanchez-Guerrero J, AlarconSegovia D. Measurement of disease activity in systemic lupus erythematosus: prospective validation of 3 clinical indices. J Rheumatol. 1992;19:1551-1558.

11. Townsend D, Jakoby B, Long M, et al. Performance and clinical workflow of a new combined PET/CT scanner [abstract]. J Nucl Med. 2007;48(suppl): $437 \mathrm{P}$

12. Nagamachi S, Czernin J, Kim AS, et al. Reproducibility of measurements of regional resting and hyperemic myocardial blood flow assessed with PET. J Nucl Med. 1996;37:1626-1631.

13. Hutchins GD, Schwaiger M, Rosenspire KC, et al. Noninvasive quantification of regional blood flow in the human heart using N13 ammonia and dynamic positron emission tomographic imaging. J Am Coll Cardiol. 1990;15:10321042

14. Siegrist PT, Gaemperli O, Koepfli P, et al. Repeatability of cold pressor testinduced flow increase assessed with $\mathrm{H}_{2}{ }^{15} \mathrm{O}$ and PET. J Nucl Med. 2006;47:14201426.

15. Prior JO, Schindler TH, Facta AD, et al. Determinants of myocardial blood flow response to cold pressor testing and pharmacologic vasodilation in healthy humans. Eur J Nucl Med Mol Imaging. 2007;34:20-27.

16. Alexanderson E, Rodriguez-Valero M, Martinez A, et al. Endothelial dysfunction in recently diagnosed type 2 diabetic patients evaluated by PET. Mol Imaging Biol. 2009;11:1-5.

17. Czernin J, Muller P, Chan S, et al. Influence of age and hemodynamic on myocardial blood flow and flow reserve. Circulation. 1993;88:62-69.

18. Kozora E, Ellison MC, West S. Depression, fatigue, and pain in systemic lupus erythematosus (SLE): relationship to the American College of Rheumatology SLE neuropsychological battery. Arthritis Rheum. 2006;55:628-635.

19. Prior JO, Quinones MJ, Hernadez-Pampaloni M, et al. Coronary circulatory dysfunction in insulin resistance, impaired glucose tolerance, and type 2 diabetes mellitus. Circulation. 2005;111:2291-2298.

20. Herzog BA, Husmann L, Valenta I, et al. Long-term prognostic value of ${ }^{13} \mathrm{~N}$ ammonia myocardial perfusion positron emission tomography: added value of coronary flow reserve. J Am Coll Cardiol. 2009;54:150-156. 